\title{
Trends in aetiology, treatment and complications associated with diabetic ketoacidosis (DKA) - a 6-year study at a large tertiary care centre in the West Midlands, United Kingdom
}

\author{
Authors: Dengyi Zhou, ${ }^{\mathrm{A}}$ Lucretia Thomas, ${ }^{\mathrm{A}}$ Eka Melson, ${ }^{\mathrm{B}}$ Andrii Kolesnyk, ${ }^{\mathrm{C}}$ Agnes Johnson, ${ }^{\mathrm{A}}$ Sandip Ghosh, ${ }^{\mathrm{C}}$ \\ Parth Narendran ${ }^{C}$ and Punith Kempegowda ${ }^{C}$
}

\section{Introduction}

Diabetic ketoacidosis (DKA) is an acute endocrine emergency that requires immediate diagnosis and management. Effective management of DKA in accordance with national guidelines improves clinical outcomes and may reduce long hospital stays. ${ }^{1}$ Recent studies have shown that frequent audit cycles, performance feedback and regular feedback of specific clinical criteria are necessary to sustain improvements in DKA management. ${ }^{2,3}$

The study has three aims.

> To identify the common aetiologies of DKA, alongside auditing the practice of including DKA as a diagnosis on this cohort's discharge letters.

$>$ To study the appropriateness of monitoring of blood glucose (BG) and ketones and of the prescription of fixed rate intravenous insulin infusion (FRIII) and fluids during DKA.

> To study the frequency of hypokalaemia, hyperkalaemia and hypoglycaemia during DKA management.

\section{Materials and methods}

All patients who were prescribed FRIII from April 2014 to August 2019 were included in the study. These episodes were then manually confirmed to meet the criteria for DKA (serum glucose $\geq 11 \mathrm{mmol} / \mathrm{L}$, ketones $\geq 3 \mathrm{mmol} / \mathrm{L}$ and $\mathrm{pH}$ $\leq 7.3$ or bicarbonate $\leq 15 \mathrm{mmol} / \mathrm{L}$ ). Data were collected regarding aetiology of DKA, duration of DKA, inclusion of DKA as a diagnosis on discharge letters and appropriateness of prescription of FRIII and fluids and of BG and ketone measurements for all episodes. Data regarding hypokalemia (potassium $(\mathrm{K})<3.5 \mathrm{mmol} / \mathrm{L})$, hyperkalemia $(\mathrm{K}>5.5 \mathrm{mmol} / \mathrm{L})$

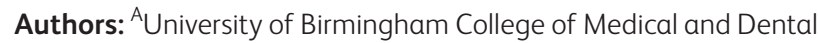

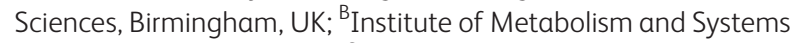
Research, Birmingham, UK; ' University Hospitals Birmingham NHS Foundation Trust, Birmingham, UK and hypoglycaemia (BG $<4 \mathrm{mmol} / \mathrm{L}$ ) were recorded for episodes from April 2018 to August 2019. DKA resolution was defined by the standard criteria (serum glucose $<11 \mathrm{mmol} / \mathrm{L}$, ketones $<0.6$ $\mathrm{mmol} / \mathrm{L}$ and $\mathrm{pH}>7.3$ or bicarbonate $>15 \mathrm{mmol} / \mathrm{L}$ ). All data were collected from the time of DKA diagnosis until resolution or up to 12 hours from diagnosis (whichever was longest).

\section{Results and discussion}

A total of 625 episodes were included in the study. The mean DKA duration was 18.6 hours. The common aetiologies leading to DKA were intercurrent illness (30.08\%), suboptimal compliance with diabetes therapy (27.2\%), new presentation of type 1 diabetes $(8.32 \%)$, surgical-related aetiologies $(6.4 \%)$, sepsis (5.44\%) and alcohol-related aetiologies (3.68\%). 17.92\% of cases had unclear aetiology. Only $69.2 \%$ of patients from April 2018 to August 2019 had the diagnosis of DKA included in their hospital discharge letter. Prescription of FRIII and fluids was appropriate in $98.2 \%$ and $84.1 \%$ of cases respectively. $63.7 \%$ of patients received the recommended hourly ketone monitoring whereas glucose monitoring was carried out more frequently than hourly, on average every 44 minutes. Only $63.1 \%$ had at least one potassium measurement during DKA. Of these, 9.6\% had hypokalemia, $17.6 \%$ had hyperkalemia and $25.3 \%$ had hypoglycaemia.

\section{Conclusion}

Nearly 70\% DKA episodes had preventable aetiology, highlighting the importance of patient education regarding diabetes management. Nearly a third of confirmed DKA cases did not have the diagnosis of DKA included in their hospital discharge letter, suggesting a need for improvement to enable follow up in primary care. Higher than recommended frequency of glucose measurements may indicate potential resource wastage, whereas under-monitoring of ketones might prolong DKA duration as it is one of the resolution criteria. High rates of kalaemic and glycaemic complications associated with suboptimal monitoring during DKA management need to be addressed urgently. Further studies are required to assess 
the impact of complications on overall DKA management, DKA duration and length of hospital stay.

\section{Conflicts of interest}

None declared.

\section{References}

1 Savage MW, Dhatariya KK, Kilvert A et al. Joint British Diabetes Societies guideline for the management of diabetic ketoacidosis. Diabet Med 2011;28:508-15.
2 Kempegowda P, Chandan JS, Coombs B et al. Regular performance feedback may be key to maintain good quality DKA management: results from a five-year study. BMJ Open Diabetes Res Care 2019;7:e00695.

3 Kempegowda P, Coombs B, Nightingale P et al. Regular and frequent feedback of specific clinical criteria delivers a sustained improvement in the management of diabetic ketoacidosis. Clin Med 2017;17:389-94. 